\title{
Hyperspectral Imaging Infrared Sensor Used for Enviromental Monitoring
}

\author{
M. Kastek ${ }^{a, *}$, T. Piatkowski ${ }^{a}$, M. Zyczkowski $^{a}$, M. Chamberland $^{b}$, P. Lagueux $^{b}$ \\ AND V. FARLEY ${ }^{b}$ \\ ${ }^{a}$ Institute of Optoelectronics, Military University of Technology, S. Kaliskiego 2, 00-908 Warsaw, Poland \\ ${ }^{b}$ Telops Inc., Quebec, Canada
}

This paper presents detection, identification and quantification of gases using an infrared imaging Fourier-transform spectrometer. The company Telops has developed an imaging Fourier-transform spectrometer instrument, Hyper-Cam sensor, which is offered as short or long wave infrared sensor. The principle of operation of the spectrometer and the methodology for gases detection, identification and quantification has been shown in the paper, as well as theoretical evaluation of gases detection possibility. The variation of a signal reaching the imaging Fourier-transform spectrometer caused by the presence of a gas has been calculated and compared with the reference signal obtained without the presence of a gas in the imaging Fourier-transform spectrometer field of view. Some result of the detection of various types of gases has been also included in the paper.

DOI: $10.12693 /$ APhysPolA.124.463

PACS: 07.57.Ty, 78.20.Ci, 78.40.-q, 78.47.jg

\section{Introduction}

A problem of remote detection of chemical substances appears in many, sometimes extremely different fields of human activities. Applications of detection devices include monitoring of technological processes, diagnostics of industrial installations, monitoring of natural environment, and military purposes. Such diversity has caused development of many detection methods employing various physical phenomena due to which detection and identification of chemical compounds are possible. Among these methods, a significant group constitutes the solutions using a phenomenon of selective absorption of electromagnetic radiation by chemical compounds. Here, we can distinguish the solutions based on absorption of laser radiation emitted by an illuminator, being an element of a measuring system, and thermovision methods recording thermal radiation. The methods of the first group are the active methods employing absorption of laser radiation by chemical compounds. Thermovision methods are based on using the absorption bands of chemical compounds in IR.

Thermovision methods, employing arrays detectors, provide an image (in real time) of the observed scenery with the marked regions of searched chemical substances. Usefulness of this method results from the fact that a lot of substances and chemical compounds have their absorption bands in IR. Figure 1 shows absorption bands of the selected chemical compounds.

The devices operating in IR, used for detection of chemical substances (gases) in the atmosphere have spec-

*corresponding author; e-mail: mkastek@wat.edu.pl
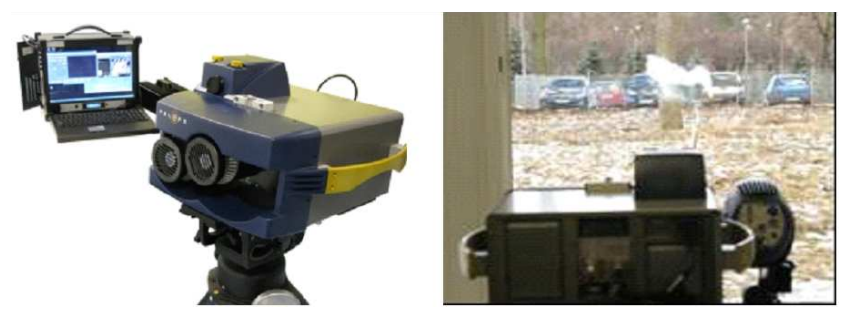

Fig. 1. The infrared IFTS - Hyper-Cam.

tral characteristics and resolution matched to the absorption bands of compounds to be detected. Two types of such devices can be distinguished. One, it is a system similar to typical thermal camera but additionally equipped with the filter system ensuring the required spectral resolution and the signal analysis system. The other type includes the device based on the principles of the Fourier spectroscopy (Fourier transform infrared spectroscopy - FTIR) that is expensive and so not readily available.

Fourier-transform spectrometers (FTS) are renowned instruments, particularly well-suited to remotely provide excellent estimates of quantitative data. Many authors have presented how they are using conventional (non-imaging) FTS to perform quantification of distant gas emissions. Amongst others, we should mention the important contributions made by Harig et al. [1, 2]. His group performed many measurement campaigns for which they obtained excellent results by ensuring proper modeling of the scene and by paying attention to understand (and to take into account) the instrument signature. Other groups developed similar approaches however limiting their study to optically thin plumes $[3,4]$ to dedicated instruments performing optical subtraction 
$[5,6]$, or introducing Bayesian algorithms to maximize the use of a priori information [7]. On the other hand, detection and quantification activities with an imaging Fourier-transform spectrometer (IFTS) have been first presented by Spisz et al. [8].

The present paper deals with the remote gas identification and quantification from turbulent stack plumes with an IFTS. It presents first the modeling that is required in order to get an appropriate understanding of both the scene and the instrument. Next it covers the methodology of the developed quantification approach. Finally, results are presented to demonstrate the capabilities and the performances of the remote gas quantification by using hyperspectral data obtained from the Telops HyperCam. The latter is described in much detail in prior Refs. [9-12].

\section{Imaging Fourier transform spectroradiometer}

The infrared IFTS - Hyper-Cam LWIR - used in experiment was built by Telops Inc. This IFTS use $320 \times 256$ pixels mercury cadmium telluride (MCT) focal plane arrays (FPA) with a $6^{\circ} \times 5^{\circ}$ FOV. The FPA has Stirling cooler to provide good noise figures in a field ready package. Spectral information is obtained using a technique called Fourier transform infrared radiometry (FTIR). FTIR is a classical interference based technique applied to gas spectroscopy that uses a Michelson interferometer to mix an incoming signal with itself at several different discrete time delays. The resulting time domain waveform, called an interferogram, is related to the power spectrum of the scene through the Fourier transform. An interferogram for each pixel in an image is created by imaging the output of the interferometer onto a focal plane array and collecting data at each discrete time delay. Advantages of using an FTIR sensor over filter or grating based system include higher resolution for equal cost and the absence of misalignment of different color images due to platform motion. However, FTIR does have the disadvantage of producing a slower frame rate than filter based systems because twice as many points are taken for the same number of spectral points. For atmospheric tracking of gases however, the LWIR sensor has sufficient frame rate [13].

Data for this collection can be taken from $0.25 \mathrm{~cm}^{-1}$ to $150 \mathrm{~cm}^{-1}$ spectral resolution between $830 \mathrm{~cm}^{-1}(12 \mu \mathrm{m})$ and $1290 \mathrm{~cm}^{-1}(7.75 \mu \mathrm{m})$ at a frame rate of $0.2 \mathrm{~Hz}$. In addition to the infrared data, visible imagery was taken using a firewire camera that is boresighted to the IR sensor. Figure 1 shows a picture of a Hyper-Cam LWIR. The sensor is controlled by field computer and data is stored on a RAID drive to guarantee integrity of data [13].

The main difference between the standard and imaging interferometer is the application of FPA detector type. The imaging device can be described as classical interferometer multiplied by the number of pixels in the array. As a result the spectral analysis of interferograms at single pixels gives the spectral information of the entire image. The spectral distribution for an entire image is obtained by performing the analysis according to (2) for every pixel of an FPA array. The results can be treated as 3-dimensional data (two image coordinates and a wavelength) and can be analysed using the special software for detection and identification of gases.

Imaging Fourier-transform spectroradiometer - $\mathrm{Hy}$ perCam - uses the layout of the Michelson interferometer. Its schematic diagram, showing the elements responsible for the change of the length of optical path, is shown in Fig. 2.

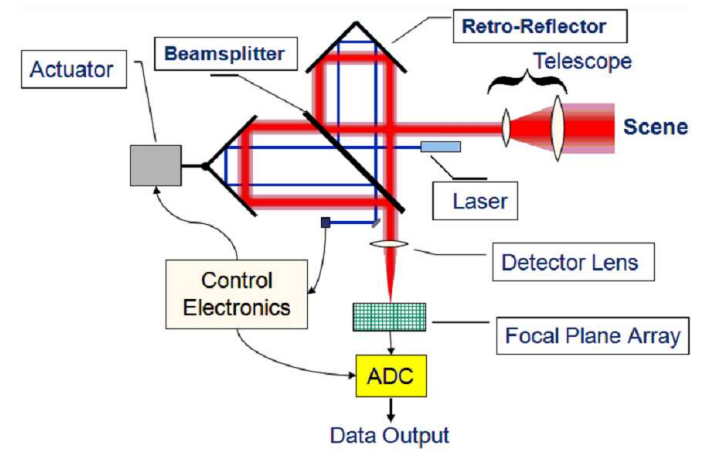

Fig. 2. Block diagram of imaging Fourier-transform spectroradiometer.

In the presented system the retro-reflectors are used instead of flat mirrors. As a result it is easier to move $\mathrm{M}_{2}$ mirror keeping the reflecting plane in constant position with respect to the optical axis. It should be mentioned here that the interferometer works in the far infrared range, thus the necessary difference in optical paths requires large mirror travel. For such long moves in a classical setup with flat mirrors the skew of mirror plane is difficult to avoid which may result in considerable measurement errors. The solution presented in Fig. 2 minimizes such errors.

The main difference between standard and imaging interferometer is the application of FPA detector type. The imaging device can be described as classical interferometer multiplied by the number of pixels in the array. As a result the spectral analysis of interferograms at single pixels gives the spectral information of the entire image. The analysis of incident radiation at pixel level is identical for every array element. The change in amplitude as a result of optical path difference $x$, for $\lambda_{i}$ wavelength is given by

$$
A(x)=A_{i} \cos \left(2 \pi \frac{x}{\lambda_{i}}\right) .
$$

Detector response is proportional to the radiant intensity of incident radiation

$$
I(t)=I_{i} \cos ^{2}\left(2 \pi \frac{x}{\lambda_{i}}\right) .
$$

Considering the trigonometric relations for the cosine of double angle and by limiting the analysis to variable component only, the radiant intensity as the function of op- 
tical path difference can be written as

$$
I(x)=I_{i} \cos \left(4 \pi \frac{x}{\lambda_{i}}\right) .
$$

For the sources with continuous radiation characteristics in the range from $\lambda_{1}$ to $\lambda_{2}$, whose spectral characteristics is described by a function $S(\lambda)$, the radiant intensity can be described as

$$
I(x)=\int_{\lambda_{1}}^{\lambda_{2}} S(\lambda) \cos \left(4 \pi \frac{x}{\lambda}\right) \mathrm{d} \lambda .
$$

Typical interferogram is shown in Fig. 3. The curve illustrates radiant intensity as the function of the translation of $\mathrm{M}_{2}$ mirror. Maximum of the output signal is achieved for identical lengths of optical paths $(x=0)$.

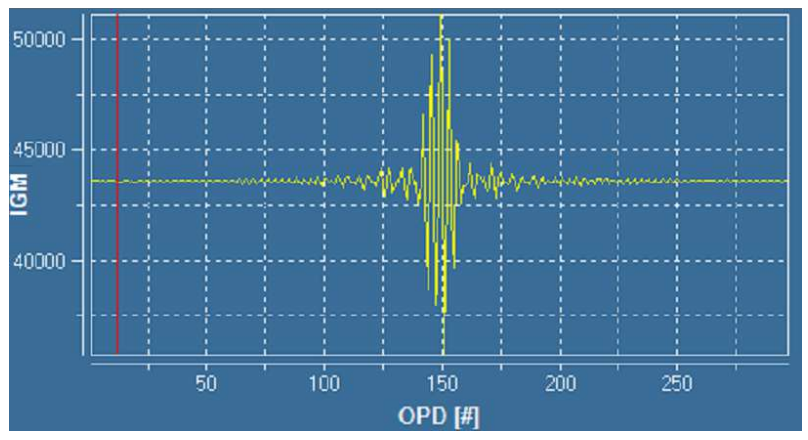

Fig. 3. Sample interferogram.

Spectral distribution for a given pixel detector can be recreated using inverse Fourier transform, by calculating the integral

$$
S(\lambda)=\int_{-\infty}^{+\infty} I(x) \cos \left(4 \pi \frac{x}{\lambda}\right) \mathrm{d} x .
$$

In practical applications the optical path difference is realized in the finite, symmetrical range $\pm d$, so the relation (5) assumes the following form:

$$
S(\lambda)=\int_{-d}^{+d} I(x) \cos \left(4 \pi \frac{x}{\lambda}\right) \mathrm{d} x .
$$

The spectral distribution for an entire image is obtained by performing the analysis according to (6) for every pixel of an FPA array. The results can be treated as 3 -dimensional data (two image coordinates and a wavelength).

The device described above is an imaging Fourier-transform infrared spectrometer. Such devices have several advantages. One of the most important parameter of any spectrometer is the product of its resolution and transmission. This coefficient is smallest for prism-type spectrometers. Grating-type spectrometers have this coefficient larger by several percent, whereas Fourier-transform devices boast up to two orders of magnitude advantage. It is particularly important in the infrared range, where the radiant intensity levels are usually much lower compared with visual range. The resolution of Fourier-transform spectrometers is comparable with grating-type counterparts. Further improve- ment can be achieved by applying longer optical path differences and by increasing the number of measurement points. Unfortunately both methods lead to longer measurement times, which are still shortest in case of Fourier-transform spectrometers than in any other type. The advantage of imaging spectrometer over scanning ones is that for a given optical path difference the entire image is analyzed simultaneously. Another advantage of the devices using the Michelson interferometer layout is quite large field of view. The telescope placed in front of the interferometer (Fig. 2) is optional and it is used only for the analysis of object having small angular dimensions.

Summing things up, there are three ways to obtain imaging spectroradiometer: thermal camera and a set of optical filters, thermal camera with a tunable filter or Fourier-transform device described above. Simple "camera plus filters" setup has the worst parameters of these three. It has fixed imaging windows defined by the number of filters (usually not more than eight). Its operation is slow because filters are mechanically inserted into optical path, e.g. by a rotation of a filter wheel. However it is a simple and cost-effective solution which can be used when the application does not require high spectral resolution, as in the detection of previously-selected gases in the chosen absorption band.

The described devices are referred to as spectroradiometers, but sometimes the more general spectrometer term is used. In case of prism or grating-type spectrometers the functional fragment called monochromator can be distinguished. Such devices can be used as sources of quasi-monochromatic radiation. This cannot be achieved with Fourier-transform spectroradiometers.

\section{Method of gas detection}

The target detection and identification algorithm is generally based on three key factors: the composition of the analyzed pixel, the type of model used to estimate the variability of the target and background spaces, and the model used to describe the pure and mixed pixels. The mathematical representation of a mixed pixel depends on whether the background (or target) space is estimated statistically or geometrically. The considered sub-pixel target detection algorithms are of a stochastic nature. When the background is entirely represented by its statistics, the detection problem consists in extracting the targeted spectral signatures from a background noise term: $\varepsilon_{\mathrm{b}}$. Equations (7) and (8) express this symbolically

$$
\begin{aligned}
& H_{0}: x=\varepsilon_{\mathrm{b}}, \\
& H_{1}: x=T_{a}+\varepsilon_{\mathrm{b}} .
\end{aligned}
$$

Here, $a$ is a weighting vector to be estimated and $T$ is a matrix of " $k$ " targets: $\left[t_{1}, t_{2}, t_{3}, \ldots, t_{k}\right]$. It is assumed that the noise component has a mean value of zero and covariance $C$.

The basic idea behind the clutter-matched filter (CMF) is to minimize the response to the unknown background signatures while accentuating the response to the target spectrum. To do so, the following mathematical 
operator was developed:

$$
q_{i}=\frac{C^{-1} t_{i}}{\sqrt{t_{i}^{\prime} C^{-1} t_{i}}},
$$

where $q_{i}$ is the CMF related to the $i$-th target $t_{i}$ taken from the matrix of targets $T$ and the $N \times N$ estimated covariance matrix, $C$, is given by Eq. (10):

$$
C=\frac{1}{M-1} \sum_{i=1}^{M}\left(x_{i}-\mu\right)\left(x_{i}-\mu\right)^{\prime} \text {. }
$$

Here, $x_{i}$ and $\mu$ are, respectively, the spectrum of the $i$-th pixel and the mean pixel of the scene and $M$ is the number of pixels from the image used to estimate the covariance. This model falls under the stochastic methods category.

The signal to clutter ratio is simply calculated with Eq. (11):

$$
\frac{\text { Signal }}{\text { Clutter }}=q\left(x_{i}-\mu\right) \text {. }
$$

If the CMF is not calculated for each scene, which is sensible if real-time processing is required, then the mean pixel found in Eq. (10) must be the one introduced in Eq. (11) when the covariance matrix was introduced.

The CMF is normalized so that, when the signal is absent, the probability density function of the filtered image has a standard deviation of 1 . The score of each pixel denotes the number of standard deviations that this pixel parts from zero. As can be ascertained with Eq. (9), the response of the CMF is linearly dependent on the target signature. The results obtained with this model give an approximate concentration of the targeted species occupying the pixels. Effectively, the scores obtained when the CMF is applied are a good indicator of the strength of the signal, which, in turn, is proportional to the temperature contrast and to the column density of the target.

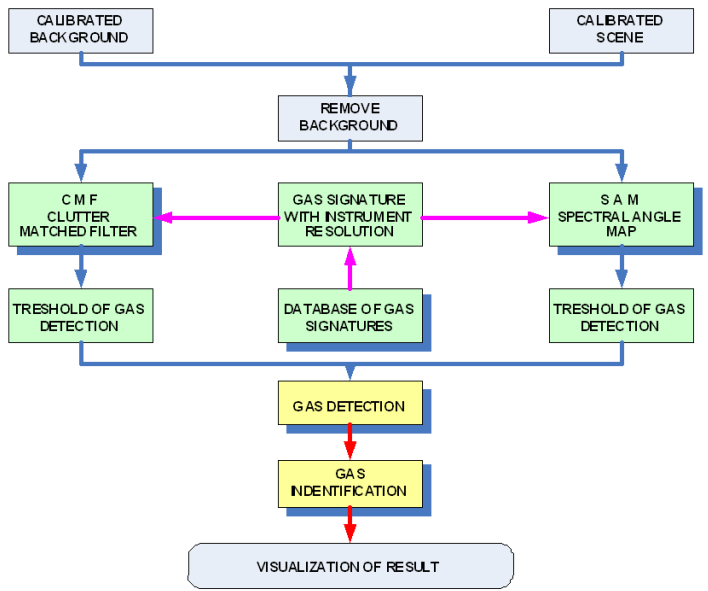

Fig. 4. Double-stage detection and identification of scheme used to process hyperspectral data.

When the target mean is known, it is possible to use the spectral angle mapper (SAM, or, as it will sometimes be called in this article, the correlation coefficient) to detect the presence of targeted species. As its name indicates, SAM is an algorithm that computes the angle formed by the projection of a target on an image pixel

$$
x_{i} t=\left\|x_{i}\right\|\left\|t_{i}\right\| \cos (\theta)
$$

or, in a more common form

$$
\cos (\theta)=\frac{x_{i} t}{\left\|x_{i}\right\|\|t\|} .
$$

The results are limited between 0 and 1 . A value of 0 signifies that the image pixel and the target are not at all correlated whereas a score of 1 means that the two vectors are perfectly correlated. SAM is obviously not a linear filter and it cannot indicate the strength of the signal. The detection and identification of chemical agent is performed through post-processing of the recorded hyperspectral. The algorithm integrates a double-stage decisional scheme used to analyze the hyperspectral data produced by Hyper-Cam sensor. The algorithm is presented in Fig. 4. Measurement of the background is performed to obtain the covariance matrix of the scene. This covariance is used to calculate the clutter-matched filters for several gas signatures from a library based on Pacific Northwest National Laboratory (PNNL) database. On each scene measurement, the background is removed and the calculation of the image of clutter-matched filter scores and correlation (SAM) scores is performed. To detect and identify a given chemical agent, the score from both calculations (CMF and SAM) must exceed a given threshold [14-16].

\section{Field tests results}

During the field tests the following gases were measured: $\mathrm{CO}_{2}$, propane-butane mixture, and freon 134 $\left(\mathrm{CH}_{2} \mathrm{FCF}_{3}\right.$ tetrafluoroethane). In each case gases concentrations were tested in order to verify the efficiency of gas measurements in the open space by HyperCam LWIR.

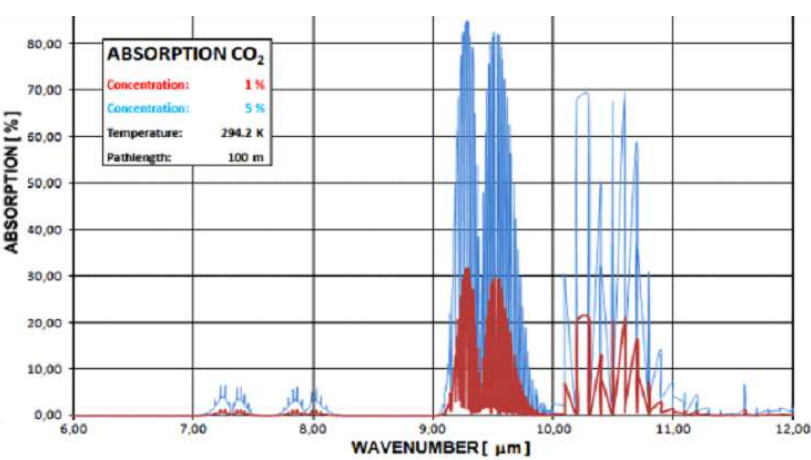

Fig. 5. The theoretical absorption $\mathrm{CO}_{2}$ for different concentration $1 \%$ and $5 \%$.

Theoretical absorption spectra for two concentrations of $\mathrm{CO}_{2}(1 \%$ and $5 \%)$ were calculated by HITRAN software and are presented in Fig. 5. As expected, higher gas concentration significantly increases the absorption 
and broadens its spectral range. During the experiment measured absorption characteristics of $\mathrm{CO}_{2}$ for two concentrations were measured, too. Freon 134 was another gas used during the field measurements. This gas exhibits absorption bands in long wave infrared range, thus it can be detected using Hyper-Cam LWIR. The registrations were made at two distances: $60 \mathrm{~m}$ and $10 \mathrm{~m}$, for two gas concentrations of $3 \%$ and $6 \%$. During the field measurements the weather conditions (humidity, pressure, wind speed, and direction) were monitored by an automatic weather station Vantage Pro. It was observed that wind speed has the most significant influence on the measurement results. The results are presented in Fig. 6a and Fig. 7a. The Matlab procedure applied for the analysis of data from Hyper-Cam utilized the reference signature database and CMF module [7].

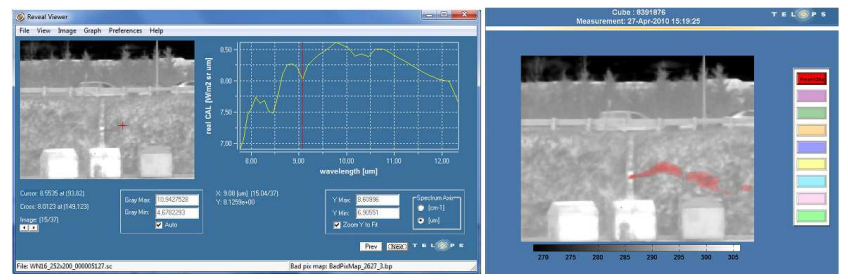

Fig. 6. The results of measure of freon 134 (concentration 3\%) at distance $60 \mathrm{~m}$ (left), freon 134 results of analysis in Matlab (right).

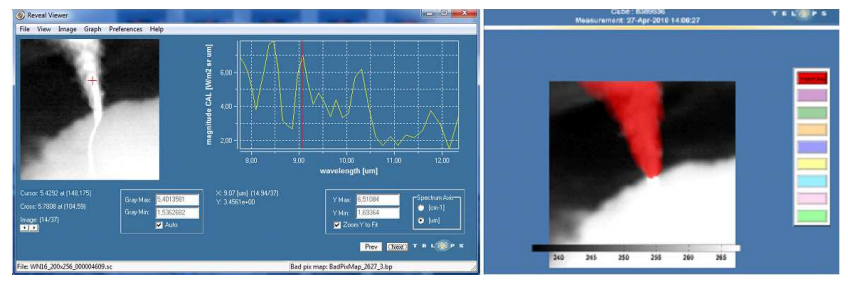

Fig. 7. The results of measure of freon 134 (concentration $6 \%$ ) at distance $10 \mathrm{~m}$ (left), freon 134 results of analysis in Matlab (right).

\section{Conclusion}

IFTS technique is a very useful method for gas detection in the open space. Its sensitivity is of order of magnitude lower than typical laboratory methods, mainly due to the fact that lock-in detection is not possible. However the possibility to present the results in a form of an image is a clear advantage, because the gas can be not only detected, but also located in space. Even its lower accuracy is still sufficient to detect and identify particular gases in the mixed gas cloud. On the basis of conducted experiments it can be stated that the gas concentrations exceeding $2 \%$ can be detected in the open space using the Hyper-Cam sensor.

After the analysis of result tests field efficiency of the IFTS technology was confirmed. During the experiment measurement of characteristics of gases were made with different conditions (different concentration) and during different environments conditions. For the over side the results of tests confirmed possibility to use this kind of spectroradiometers to automatical detection of gases, after the development method and software for it.

The measurements conducted by means of IFTS spectrometers have lower accuracy due to lower sensitivity, the influence of weather conditions (mainly wind speed) and unknown thickness of a visualized gas cloud. Currently the new quantification algorithm is under development, in which the cloud thickness will be automatically estimated on the basis of spatial distribution of areas with emissivity different than that of a background. This new method combining mathematical analysis, data from reference signature database (PNNL database), CMF module and additionally SAM module should improve the effectiveness of gas detection by IFTS-based solutions.

\section{References}

[1] R. Harig, G. Matz, Field Anal. Chem. Technol. 5, 75 (2001).

[2] R. Harig, G. Matz, P. Rusch, Proc. SPIE 4574, 83 (2002).

[3] M.K. Griffin, J.P. Kerekes, K.E. Farrar Burke, K.H.H., Proc. SPIE 4381, 360 (2001).

[4] T. Burr, N. Hengartner, Sensors 6, 1721 (2006).

[5] R.L. Lachance, J.-M. Thériault, C. Lafond, A.J. Villemaire, Proc. SPIE 3383, 124 (1998).

[6] J.-M. Thériault, Technical Report Defence Research Establishment Valcartier (DREV) TR-2000156 (2001).

[7] P. Heasler, C. Posse, J. Hylden, K. Anderson, Sensors 7, 905 (2007).

[8] T.S. Spisz, P.K. Murphy, C.C. Carter, A.K. Carr, A. Vallières, M. Chamberland, Proc. SPIE 6554, 655408 (2007).

[9] V. Farley, M. Chamberland, P. Lagueux, A. Vallières, A. Villemaire, J. Giroux, Proc. SPIE 6661, 66610L (2007).

[10] A. Vallières, A. Villemaire, M. Chamberland, L. Belhumeur, V. Farley, J. Giroux, J.-F. Legault, Proc. SPIE 5995, 59950G (2005).

[11] M. Chamberland, C. Belzile, V. Farley, J.-F. Legault, K. Schwantes, Proc. SPIE 5416, 63 (2004).

[12] V. Farley, C. Belzile, M. Chamberland, J.-F. Legault, K. Schwantes, Proc. SPIE 5546, 29 (2004).

[13] M. Kastek, T. Piątkowski, P. Trzaskawka, Metrol. Measur. Syst. 18, 679 (2011).

[14] P. Tremblay, S. Savary, M. Rolland, A. Villemaire, M. Chamberland, V. Farley, Proc. SPIE $\mathbf{7 6 7 3}$ $76730 \mathrm{H}$ (2010).

[15] M. Kastek, T. Piatkowski, R. Dulski, M. Chamberland, P. Lagueux, V. Farley, Phot. Lett. Poland 4, 146 (2012).

[16] M. Kastek, T. Piatkowski, R. Dulski, M. Chamberland, P. Lagueux, V. Farley, in: Symp. on Photonics and Optoelectronics SOPO 2012, IEEE Photonics Society, Shanghai 2012, art. no. 6270545. 